\title{
Ageing across the Tasman Sea: the demographics and health of older adults in Australia and New Zealand
}

\author{
Paul Kowal, ${ }^{1,2}$ Andy Towers, ${ }^{3}$ Julie Byles ${ }^{2}$
}

tos he prospect of demographic ageing has been followed closely over past decades in Australia and New Zealand (NZ). These two countries, separated by the Tasman Sea, are members of the Organization for Economic Cooperation and Development (OECD), are similar in terms of age structure and the rate of population ageing, and face similar issues regarding the need to provide health and social policy for an ageing population. Both stand out within the Oceania region of Australia/NZ, Melanesia, Micronesia and Polynesia' in terms of population ageing, with around $15 \%$ aged 65 years and over, compared to less than $10 \%$ in all other countries in the region. ${ }^{2}$

The growth of the older adult population in Australia has directly paralleled NZ over the past half century, the proportion aged 65 years or older in both countries having risen from about $9 \%$ of the total populations in 1960 to $15 \%$ in $2013 .{ }^{2}$ In both countries, population ageing is well progressed and the respective rates of ageing will increase steadily through the year 2100 . The period between 2005 and 2040 sees a more rapid rate of ageing in NZ than in Australia, resulting in a comparatively higher percentage of the population $65+$ from about 2025 , with the rates more or less the same for the countries from 2040 carrying through to 2100. For Australia and NZ, life expectancies at birth have increased by more than eight years for males and six years for females over the past 30 years; with current life expectancy nearing 80 years for Australian and NZ men (80.1 and 79.1 years, respectively) and 83

\begin{abstract}
Objective: The demographic and health aspects of ageing populations in Australia and New Zealand (NZ) are described. These data are relevant to compare impacts of policy and context in each country.

Methods: Secondary analysis of international (Organization for Economic Co-operation and Development, United Nations and World Health Organization) and domestic population and health data.

Results: Both countries will experience a greater than $80 \%$ increase in the population aged 60-plus years between 2013 and 2050. The increase in the 80 -plus population will be $200 \%$ or higher, resulting in 2.8 million Australians and more than 510,000 New Zealanders in this age group by 2050 . The speed of ageing in both countries is higher than the average rate of increase in developed countries. Average life expectancy at birth and age 60 is higher in Australia than NZ, with the differences increasing slightly by 2050, and gaps between men and women consistently smaller in NZ than in Australia. However, a higher proportion of older Australians report living with a disability (53\%) than older New Zealanders (45\%).

Conclusions: Australia and NZ are well aged in the context of a youthful Oceania region, with more similarities than differences between the countries.

Implications: Both countries need to continue to monitor health trends, unravel the major population attributable risks, and identify preventative and other interventions that can stimulate and support declines in disability in older populations in the future, particularly for non-indigenous older persons.
\end{abstract}

Key words: ageing, demographic, life expectancy, disability, gender

years or more for women $(84.7$ and 82.9 years, respectively). ${ }^{2}$ In addition to the certainty that populations are ageing, the speed of ageing matters because of the resulting need for governments and societies to adapt quickly in the coming decades.

From a health services perspective, larger numbers of people over 60 , and particularly over 85 , will require a refocusing of the care systems. Demand for services will be driven by chronic diseases that contribute an ever expanding portion of morbidity and mortality. Moreover, most older people will have multiple conditions affecting more than one body system, requiring a move away from the prevailing disease-based model of health service delivery. Preventive approaches will need to prioritise common risk factors for chronic disabling conditions, not just those that have impact on mortality.

Policymakers and researchers are keen to predict the impacts of ageing on health and social services, labour force participation, income and retirement policies - then to

\footnotetext{
1. World Health Organization Study on global AGEing and adult health (SAGE), Switzerland

2. University of Newcastle Research Centre on Gender, Health and Ageing (Hunter Medical Research Institute), New South Wales

3. Massey University School of Health and Social Services, New Zealand

Correspondence to: Dr Paul Kowal, WHO HIS/HSI, SAGE, 20 Avenue Appia, CH-1211 Geneva 27 Switzerland; e-mail: kowalp@who.int; paul.r.kowal@gmail.com

Submitted: December 2012; Revision requested: February 2013; Accepted: November 2013

The authors have stated they have no conflict of interest.
} 
compare the impacts of different policies and interventions. Yet Trans-Tasman comparisons of ageing have been limited. ${ }^{3}$ The rationale for this paper was to synthesise the demographic and health data from Australia and NZ in order to compare their current state and potential futures with regard to population ageing. This paper summarises demographic and health data from Australia and NZ.

\section{Methods}

The United Nations Population Division data (medium-fertility variants) were used for the demographic estimates and projections. ${ }^{2,4}$ The World Health Organization's 2004 Global Burden of Disease Update and the OECD 2012 health data were compiled for the burden of disease results., ${ }^{5,6}$ Data from the Australian Bureau of Statistics and NZ's Ministry of Health, Ministry of Social Development, and Statistics NZ were sourced for the disability, health, and trends information in older indigenous populations. The authors compiled results using the most recent data from each source.

\section{Results}

\section{Demographics}

Median age is a commonly used metric for population ageing, dividing the population so that half the population is younger and half older than the median age. The median age is currently 37.4 years in Australia and 37.3 years in NZ (Figure 1). The next highest in the Oceania region is New Caledonia at 33.1, while the lowest is the Solomon Islands at 19.9 years. The median age for Australia and NZ are substantially higher than the lowest OECD country median age (Mexico at 27.7 years), similar to the US (37.7 years), and below the UK (40.5 years), Canada (40.5 years), and Japan (46.5 years). By 2050, the median age in Australia will increase to 40.6 years and in NZ to 42.3 years. This increase is similar to the UK (43.3) and US (40.6), but much less than projected for South Korea and Japan, which will both exceed 53 years by 2050 .

\section{Population aged 60 years or older}

The percentage of the total population aged 60+ in Australia surpassed those aged 0-14 years in 2010. NZ will experience the same phenomenon in 2015. As of 2013, 19.8\% of Australia's total population was aged 60 years or older. This proportion will increase steadily to $27.6 \%$ by 2050 and $35.5 \%$ by 2100 . As of 2013 in NZ, the 60+ population will reach $19.2 \%$ of the total population, and with a more rapid increase of older adults between 2005 (16.4\%) and 2035 (27.1\%) than Australia, after which the $60+$ proportion is higher in NZ (28.8\% in 2050 and $36.7 \%$ in 2100) but proportions increasing at a similar rate in the two countries through 2100. Australia will see an increase of $40 \%$ and $\mathrm{NZ}$ of $50 \%$ for this population group between 2013 and 2050 .

\section{Population aged 80 years or over}

About 894,000 people aged 80 years or older lived in Australia as of 2013 and 160,500 in NZ. ${ }^{2}$ This represents $3.8 \%$ of the total
Australian population and $3.6 \%$ of the total NZ population. The number of persons aged $80+$ years will increase $211 \%$ in Australia and 220\% in NZ between 2013 and 2050, resulting in 2.7 million Australians and 513,000 New Zealanders in this age group by the year 2050 .

\section{Speed of ageing}

The average annual population growth percentage rate for 2013 is $1.7 \%$ in Australia and $1.1 \%$ in NZ. ${ }^{2}$ The corresponding rates for the populations aged $60+$ are $2.9 \%$ and $2.8 \%$, respectively. These rates are well above the global average for total populations and older populations in developed countries $(0.3 \%$ and $1.9 \%$, respectively).

\section{Figure 1: Median age for Australia, New Zealand and a select group of countries, 1950 to 2100.}

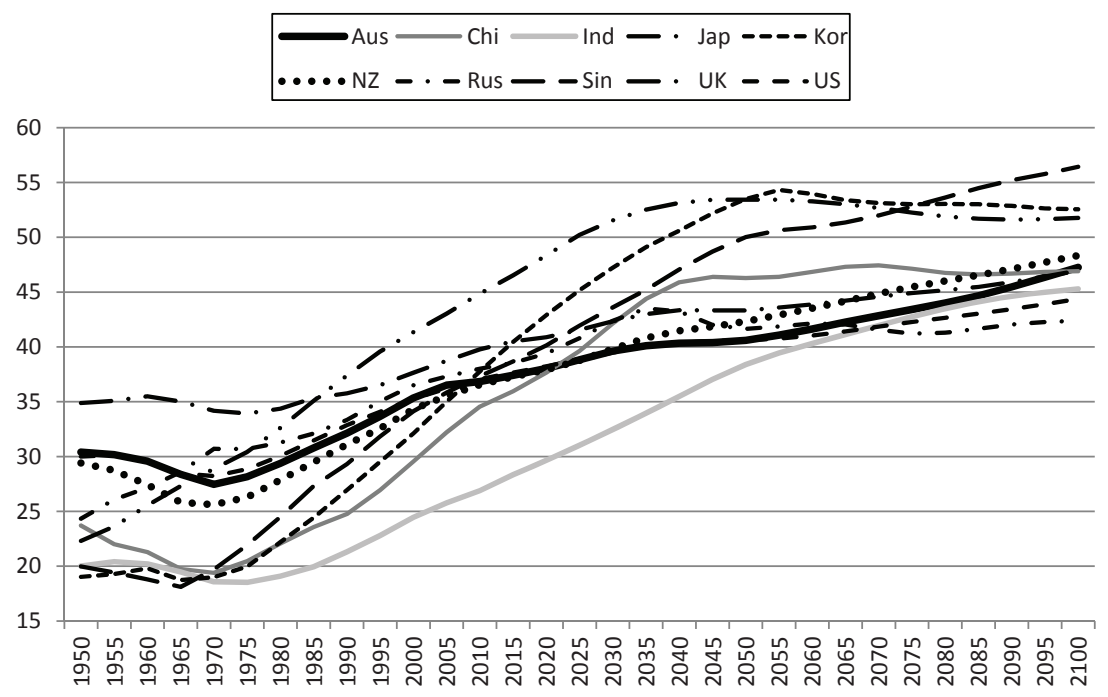

Source: United Nations, Department of Economic and Social Affairs, Population Division. World Population Prospects: The 2012 Revision. (medium-fertility variant) 2013. Aus, Australia; Chi, China; Ind, Indonesia; Jap, Japan; Kor, South Korea; NZ; New Zealand; Rus, Russian Federation; Sin, Singapore; UK, United Kingdom; US, United States.

Figure 2: Percentages of the populations aged 0 to 14 years $(0-14), 60$ years and older $(60+)$, and 80 years and older (80+) for Australia and New Zealand, 1950-2100.

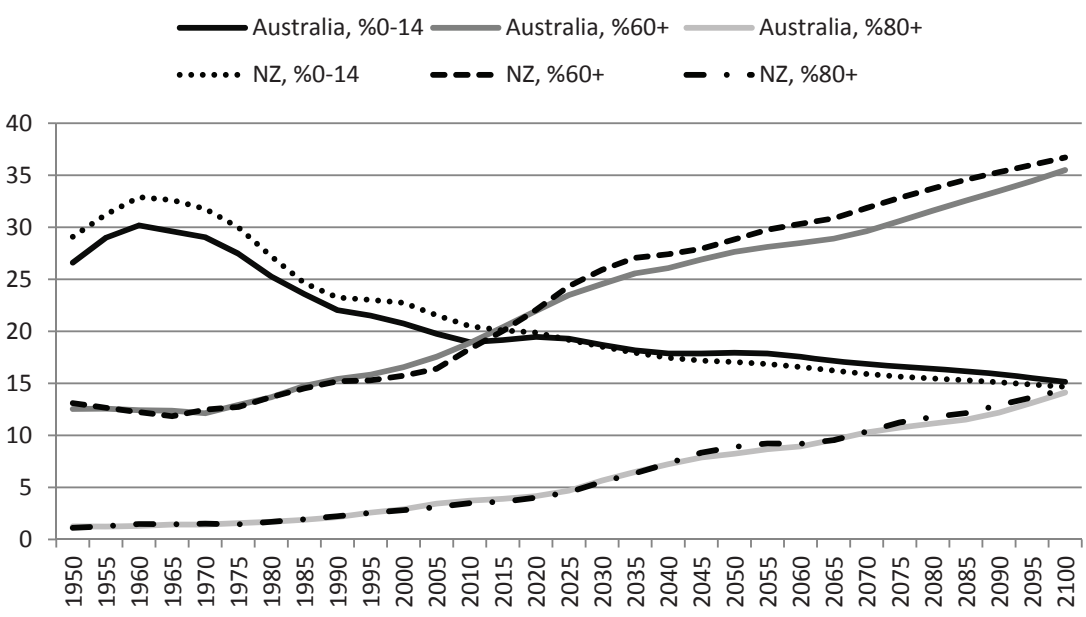

Source: United Nations, Department of Economic and Social Affairs, Population Division. World Population Prospects: The 2012 Revision. (medium-fertility variant) 2013. 
The projected trends in median age and population age-group expansion indicate that population ageing accelerates over the next two decades, reaching the highest rate of increase before 2035. After that, the speed of ageing is expected to decelerate, although there will be further increases in older populations throughout the century. These two measures of ageing suggest a significant acceleration in population ageing in both countries over the coming years.

\section{Life expectancy and healthy life expectancy}

Life expectancy at birth (LEB) in 2010 was higher in Australia than NZ (Table 1). LEB is likely to increase to 86.5 years for females and 82.4 years for males in Australia by 2030, with respective NZ LEB a little lower at 85.1 years (female) and 81.5 years (male). An Australian woman who survives to 60 years (LE60) by 2030 can expect to live another 28.3 years, reflecting almost a two year increase on 2010 estimates.

However, not all the additional years of life will be in good health. While an Australian female born in 2013 can expect to live to about 84.2 years, assuming current mortality rates prevail across the lifetime, only 74.3 years are expected to be in good health. $A$ female born in NZ can expect 72.2 years in good health, which is more than two years less than her Australian counterpart (Table 1).

\section{Life expectancy and health trends for indigenous peoples}

Indigenous populations in each country are at a distinct life expectancy disadvantage compared to non-indigenous populations. The differences in life expectancy between men and women show similar patterns in indigenous and non-indigenous populations. Among indigenous populations, LEB is higher in NZ than in Australia. The most recent LEB estimates for an Australian Aboriginal woman is around 72.9 years, while a NZ Māori woman can expect to live 75.1 years. ${ }^{7,8}$ For men, this was 67.2 years in Australia and over 70 years in NZ. ${ }^{9}$ The average difference between indigenous and non-indigenous population life expectancies are lower in NZ (8.6 years) than Australia (over 10 years). Life expectancy at age 50 is about 25 years for Māori men and 28 years for Māori women - about six years lower on average than the non-Māori population. ${ }^{10}$ For Australia, this is about 24 years for Aboriginal men and 27 years for Aboriginal women. ${ }^{11}$
A number of health risks play a significant part in health and life expectancy differences, including and quite possibly most importantly, foetal origins and social determinants of adult health. ${ }^{12-17}$ The more proximate factors, such as smoking and obesity, are contemporary risks that contribute to disease and life expectancy. Rates of smoking showed very little difference between the sexes; about $46 \%$ of Australian Aboriginal and Torres Strait Islander men were current smokers compared to $43 \%$ of women, and compared to smoking rates for Māori men (38\%) and women (44\%). ${ }^{18,19}$ While smoking rates in Australian Aboriginal people and NZ Māori have decreased in the past 20 years, both populations are still significantly more likely to smoke than their non-indigenous counterparts, 18,19 and this difference is exaggerated in older age. Older Aboriginal men and women are both almost three times more likely to be smokers as compared to non-Aboriginal people; ${ }^{18,19}$ however, older Māori men are only twice as likely as older non-Māori men to be smokers, whereas older Māori women are more than three times more likely than non-Māori women to smoke. ${ }^{10}$ Obesity is also a significant factor, with high and likely increasing rates of obesity in the indigenous populations in both countries..$^{18-21}$

\section{Women and men}

Currently, there are more women than men aged $60+$, however the male:female ratio is projected to increase over the next two decades. Further projections to 2050 indicate a greater divergence in sex ratios between the two countries (Figure 3).

Average longevity for both sexes will continue to increase through 2030, with smaller gaps between females and males in LEB projected in a number of OECD countries. However, the gap in LE60 will expand in some countries (like the US, UK and Canada) and shrink in others (like Australia and NZ).

The gap in LEB, LE60 and life expectancy at age 80 (LE80) between the sexes illustrates the continued extra years lived by women in Australia and NZ (Figure 4). For Australia, the LEB sex gap will fall through the year 2020; then level off for the next three decades. The
Figure 3: Overall sex ratios and for age groups 60+ and 80+ years for Australia and New Zealand, 1990-2100.

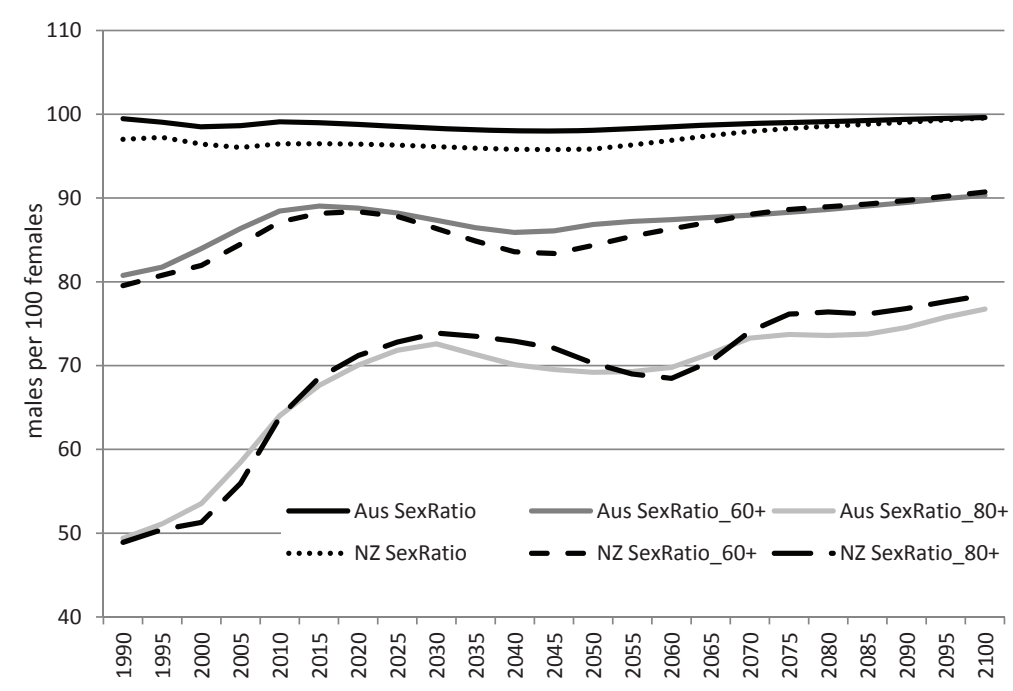

Source: United Nations, Department of Economic and Social Affairs, Population Division. World Population Prospects: The 2012 Revision. (medium-fertility variant) 2013.

Table 1: Life Expectancy (LE) and Health Adjusted Life Expectancy (HALE) (in years) by sex, Australia and New Zealand, 2010.

\begin{tabular}{|c|c|c|c|c|c|c|c|c|}
\hline & \multicolumn{4}{|c|}{ Australia } & \multicolumn{4}{|c|}{ New Zealand } \\
\hline & \multicolumn{2}{|c|}{ Female } & \multicolumn{2}{|c|}{ Male } & \multicolumn{2}{|c|}{ Female } & \multicolumn{2}{|c|}{ Male } \\
\hline & 2010 & 2030 & 2010 & 2030 & 2010 & 2030 & 2010 & 2030 \\
\hline LE at Birth & 84.2 & 86.5 & 79.8 & 82.4 & 82.7 & 85.1 & 78.8 & 81.5 \\
\hline LE at 60 years & 26.4 & 28.3 & 23.2 & 25.1 & 25.3 & 27.2 & 22.4 & 24.5 \\
\hline HALE at Birth & 74.3 & - & 70.9 & - & 72.2 & - & 69.5 & - \\
\hline HALE at 60 years & 19.5 & - & 16.9 & - & 18.2 & - & 16.0 & - \\
\hline
\end{tabular}


sex gap in LE60 shows less change, while the gap in LE80 will rise steadily - meaning that the feminisation of ageing ${ }^{22}$ will continue in Australia. NZ's sex differences in LE are overall lower than Australia and follow slightly different patterns of change. The sex gap largely declines for LEB and increases for LE80, with a shallow U-shaped pattern for LE60 between 1995 and 2050.

\section{The burden of disease and disability}

A trans-Tasman comparison of disability rates in the population aged $65+$ shows some considerable difference in patterns. About $53 \%$ of Australians aged $65+$ report living with a disability in comparison to only $45 \%$ of New Zealanders aged $65+$ years. ${ }^{23,24}$ These NZ older adult disability rates show a steady decline over the five years between surveys from 2001 to 2006 with the proportion reporting disability dropping from an original rate of 54\% in 2001. However, Australian disability incidence rates remained relatively static over this same period. ${ }^{25}$ Despite a drop in disability rates for those aged 65+, a greater proportion receive long-term care (particularly home-based) in NZ (16\%) than in Australia (14\%). ${ }^{26}$

Major contributors to death and disability burden (measured as disability adjusted life years) for older people in NZ and Australia in 2004 are shown in Figure 5. Similar patterns are seen for both countries with main causes of disability being cancer, cardiovascular disease, and Alzheimers dementia.

A comparison of causes of mortality and potential years of life lost (PYLL) for Australia and NZ (from 2006) is shown in Table 2. This data is based on the International Classification of Diseases, Tenth Revision (ICD10) and was drawn from the new OECD (2012) Health Data 2012 e-database. ${ }^{5}$ While the total number of deaths (all-cause mortality) was higher in Australia than NZ as a result of population size differences, standardised mortality rates indicate that the disease burden in NZ $(810.6$ deaths per 100,000$)$ is considerably higher than in Australia (705.6 deaths per 100,000).

The PYLL attributable to communicable diseases is less than $4 \%$ in both Australia and NZ, and the four key causes of mortality in both countries are non-communicable diseases and chronic conditions; neoplasms (cancer) and diseases of the circulatory system (ischaemic heart disease, acute myocardial infarction and cerebrovascular disease). These conditions are responsible for
$64 \%$ of deaths in Australia and $69 \%$ in NZ, so about two-thirds of mortality between these countries is directly attributable to chronic health conditions.

\section{Discussion}

Australia and NZ have similar age profiles and population ageing rates that put them ahead of other countries in the region and on par with many other major developed nations. Yet, despite demographic similarities, health and disability differences exist and point to a need for research and policy investigation. The rate of demographic change is of major public health importance not only because of the increased health needs of people at older ages, but also in relation to the changing prevalence of modifiable risk factors in earlier life that affect later life health.
The population prevalence of many noncommunicable conditions (Table 2) increase significantly with age, from key mortality indicators such as neoplasms (cancer) and diseases of the circulatory system, through to less established mortality indicators such as endocrine, nutritional and metabolic diseases and diseases of the genitourinary system. ${ }^{27,28}$ As population ageing continues in Australia and NZ, a feasible scenario would see health care utilisation levels rise, assuming static morbidity rates while taking into account levels of under-diagnosis of conditions in older age, if considerable reconfiguring of health systems do not occur. ${ }^{29-32}$

NZ experiences greater rates of mortality and years of lost life for each of these conditions, and already caters for more long-term care patients on substantially lower government health expenditure, and fewer numbers of

\section{Figure 4: Average female minus male life expectancy (LE gap) at birth, age 60 and age 80 for Australia and New} Zealand, 1950-2100.

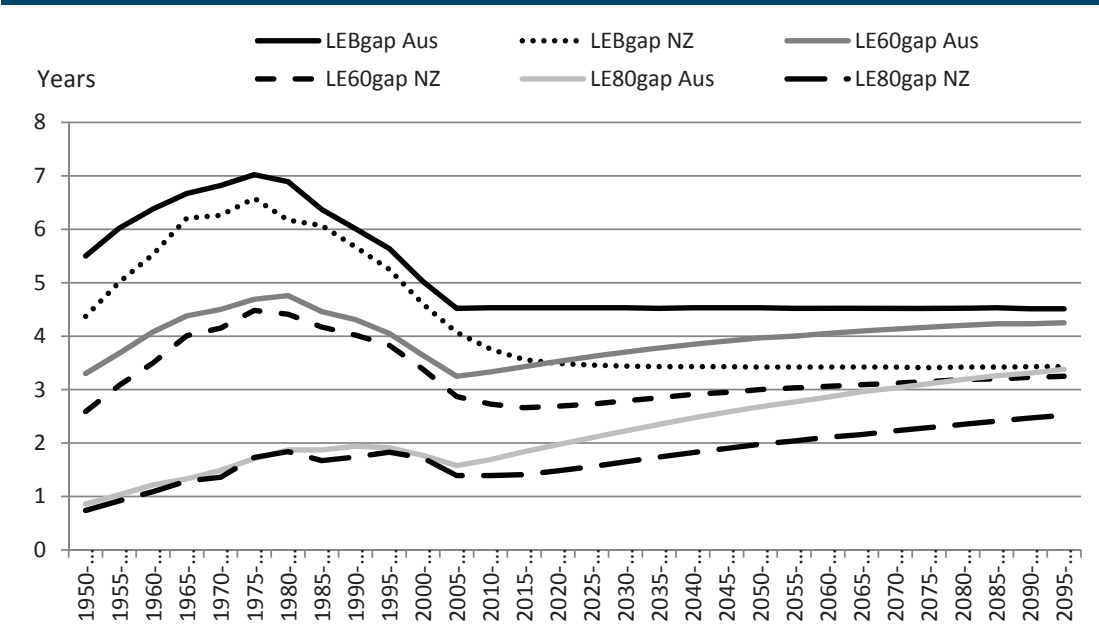

Source: United Nations, Department of Economic and Social Affairs, Population Division. World Population Prospects: The 2012 Revision. (medium-fertility variant) 2013.

\section{Figure 5: Percentage contribution to total DALYs for people 60 years or over Australia and New Zealand.}

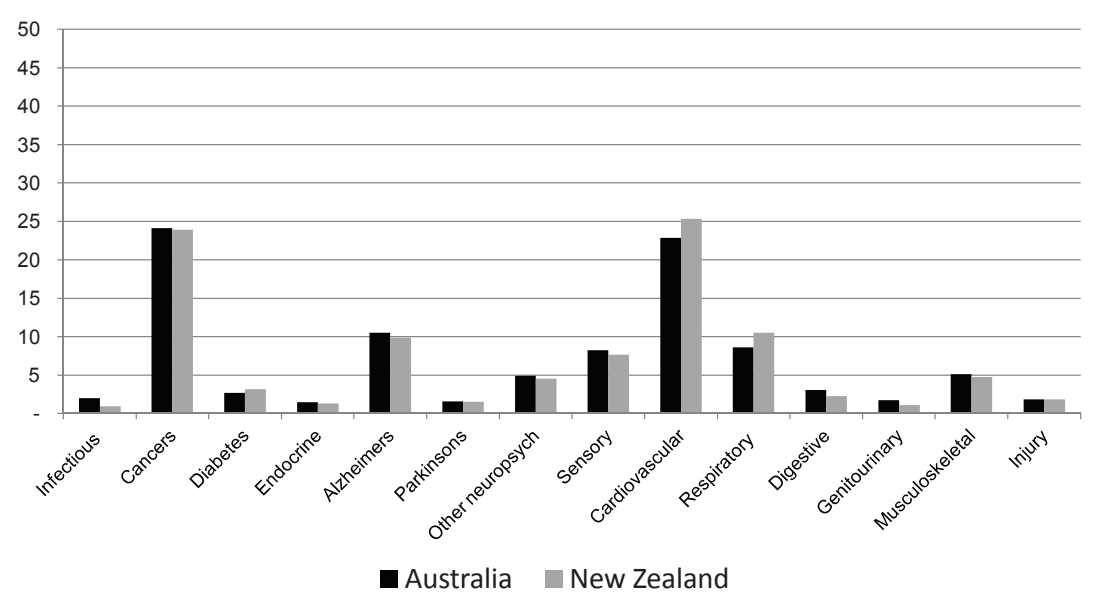

Source: WHO Global Burden of Disease Country Estimates 2004 http://www.who.int/healthinfo/global_burden_disease/estimates_country/en/index.html 
health-care staff. ${ }^{26} \mathrm{~A}$ rise in the prevalence of these conditions will likely have greater health and fiscal ramifications for NZ than Australia, although the possible impact will be tempered by NZ's more innovative and responsive social policy approaches.

NZ appears to have a lower disability burden among the older population, and greater declines in disability when compared to Australia, although contributors to disease burden estimates are similar. Similarly, reported disability rates are slightly lower in the general population in NZ (17\%) $)^{33}$ compared with Australia (19\%); ${ }_{i}^{23}$ with rates for both countries being relatively stable over the previous decade. ${ }^{25,33} \mathrm{NZ}$ has higher self-rated good health ${ }^{26}$ and happiness. ${ }^{34}$ However, NZ also has a higher mortality rate, a result of higher rates in key conditions including heart disease, stroke, cancers, and even suicide. ${ }^{26}$

In both countries, the major causes of morbidity and mortality, including cardiovascular disease, diabetes, and musculoskeletal conditions, are strongly linked to obesity levels.
Obesity rates are higher in NZ (26.5\%) than Australia (24.6\%). ${ }^{26}$ The rate of obesity in the NZ general population has been increasing exponentially over the past 20 years, while Australia's rate of increase has slowed. Both countries are likely to see increased levels of obesity-related morbidity as current cohorts enter their older years with the adverse effects of their higher body weight. ${ }^{35}$

It is still unclear whether patterns of agerelated health and functioning declines in cohorts who are now in late-middle age will differ from those of the current older cohort in each country, or how the differing levels of health and disability will influence health care costs. What is clear is that ageing, per se, will not increase health costs, and also that untreated disease or disability among older adults represents a substantial economic loss to productivity in both countries. ${ }^{36,37}$ Given Australia's currently stable disability rates, neither an expansion nor compression of morbidity has been projected in the shortterm. ${ }^{25}$ Over the same period, NZ is projected to experience a 'dynamic equilibrium' whereby the period of life lived in severe disability is decreasing but this is matched by an increase in the period of life lived with low to moderate disabilities. ${ }^{32,38}$ The effects of healthy lifestyles on subsequent disability are also large, particularly tobacco avoidance and regular exercise; however, this has been offset by increases in obesity-related disease and disability. The increasing prevalence of obesity and obesity-related conditions suggests that any compression of morbidity might be reduced, or even short-lived. ${ }^{26,31}$

The demographics of older populations will continue to be dominated by women. This disparity indicates a need for more insight into the differential determinants and drivers of health between the sexes. Higher riskfactor rates among men have been shown to have an important role in differences in longevity, ${ }^{39}$ but the excess disability among women is not well understood. Considering that women live longer than men, ${ }^{40,41}$ yet consistently report lower health levels and higher age-related disability prevalence in many countries, ${ }^{6,26}$ a clearer understanding of women's health in particular is needed. However, the health needs of older men are also under-researched.

Table 2: A comparison of the burden of disease (deaths and potential years of life lost) in Australia and New Zealand in 2006.

\begin{tabular}{|c|c|c|c|c|c|c|}
\hline & \multicolumn{3}{|c|}{ Australia 2006} & \multicolumn{3}{|c|}{ New Zealand 2006} \\
\hline & Total (\%) & Per $100,000^{b}$ & PYLLc $^{c}$ & Total (\%) & Per $100,000^{b}$ & PYLLC \\
\hline \multicolumn{7}{|l|}{ All-cause mortality ${ }^{\mathrm{a}}$} \\
\hline Male & $65,322(51.2 \%)$ & 695 & $3,702.2$ & $14,014(49.4 \%)$ & 774.5 & 4438 \\
\hline Female & $62,310(48.8 \%)$ & 705.8 & $2,086.1$ & $14,375(51.6 \%)$ & 837.9 & $2,695.2$ \\
\hline Total & $127,632(100 \%)$ & 705.6 & $2,883.9$ & $28,389(100 \%)$ & 810.6 & 3539 \\
\hline \multicolumn{7}{|l|}{ Communicable causes } \\
\hline Pneumonia & $2,603(2.0 \%)$ & 14.9 & 20.9 & $461(1.6 \%)$ & 13.9 & 20.6 \\
\hline Infectious/parasitic diseases (e.g., HIV/AIDs \& tuberculosis) & $1,868(1.5 \%)$ & 10.2 & 51.7 & $167(<1 \%)$ & 4.6 & 48.4 \\
\hline Influenza & $16(<1 \%)$ & 0.1 & 1.6 & $17(<1 \%)$ & 0.5 & 1.2 \\
\hline \multicolumn{7}{|l|}{ Non-communicable causes } \\
\hline Neoplasms (Cancer) & $38,022(29.8 \%)$ & 207.4 & 821.3 & $8,171(28.8 \%)$ & 229.4 & 984.7 \\
\hline Ischaemic heart disease & 21,940 (17.2\%) & 123.2 & 208.3 & $5,911(20.1 \%)$ & 172.9 & 299 \\
\hline Acute myocardial infarction & $10,997(8.6 \%)$ & 61.8 & 85.3 & $2,927(10.3 \%)$ & 85.5 & 141.2 \\
\hline Cerebrovascular diseases & $10,975(8.6 \%)$ & 62 & 71.8 & $2,672(9.4 \%)$ & 78.7 & 97.9 \\
\hline Endocrine, nutritional \& metabolic diseases & $4,894(3.8 \%)$ & 25.5 & 69.5 & $1,177(4.1 \%)$ & 33.1 & 155.8 \\
\hline Diseases of the nervous system (e.g., Parkinson's, Alzheimer's) & $4,693(3.7 \%)$ & 26 & 105.4 & $1,070(3.8 \%)$ & 30.8 & 123.4 \\
\hline Chronic obstructive pulmonary diseases & $4,617(3.6 \%)$ & 25.7 & 26.1 & $1,546(3.8 \%)$ & 44.9 & 57.7 \\
\hline Mental/behavioural disorders (e.g., dementia \& drug) & $4,887(3.8 \%)$ & 28 & 20.2 & $826(2.9 \%)$ & 24.8 & 19.4 \\
\hline Diseases of the digestive system & $4,290(3.4 \%)$ & 23.6 & 99.5 & $816(2.9 \%)$ & 23.5 & 69.1 \\
\hline Diseases of the genitourinary system & $3,172(2.5 \%)$ & 18 & 17.7 & $407(1.4 \%)$ & 12.1 & 20.4 \\
\hline Asthma & $389(<1 \%)$ & 2.1 & 10.6 & $79(<1 \%)$ & 2.1 & 23.5 \\
\hline Disease of blood/blood forming organs & $498(<1 \%)$ & 2.6 & 14 & $58(1<\%)$ & 1.6 & 8 \\
\hline External causes & $7,343(5.8 \%)$ & 37.8 & 730.9 & $1,744(6.1 \%)$ & 44.6 & 954.1 \\
\hline \multicolumn{7}{|l|}{ Accidents, falls, poisoning, intentional self-harm \& assault } \\
\hline $\begin{array}{l}\text { a Figures inside parentheses reflect proportion of total all-cause mortality } \\
\text { b Standardised mortality rate (that is, mortality per 100,000 population) } \\
\text { CPYLL: Potential Years of Life Lost = years of life lost per 100,000 populatic } \\
\text { Data source: OECD (2012). Health Data 2012. Available from: http://stats. }\end{array}$ & $\begin{array}{l}\text { le of } 0-69 \text { years } \\
\text { px?DataSetcode }=H E \text { E }\end{array}$ & & & & & \\
\hline
\end{tabular}


Australians are living longer and in better health than their NZ counterparts, but this trend is reversed for indigenous populations. The past two decades have seen a considerable reduction in life expectancy inequalities historically evident between NZ Māori and non-Māori. Between 1997 and 2007 Māori LEB increased almost four years for men and women, in comparison to 3.6 for non-Māori men and 2.4 for non-Māori women. ${ }^{33}$ The majority of continuous gains over this period were for Māori women, with a $15 \%$ reduction in the gap with non-Māori women, while the gap between Māori and non-Māori men has remained relatively stable. The reduction in life expectancy inequalities between Māori and non-Māori populations over this time reflects reductions in major contributors to death in Māori (e.g. cardiovascular disease and suicide) as well as a narrowing of the gap in socioeconomic status and labour market participation between Māori and non-Māori. ${ }^{42}$ The trend in Australia is not as encouraging: available evidence suggests that the large differences in health and life expectancy between indigenous and non-indigenous Australians are not shifting despite political efforts to close the gap. ${ }^{43}$ Older indigenous people in both countries are under-represented in population health studies, with more research underway in NZ than Australia to address ageing and health in these populations. ${ }^{44-48}$

NZ has a higher proportion of formal long-term care recipients than Australia. ${ }^{49}$ Per capita health expenditures using US\$ Purchasing Power Parity (PPP) were lower in NZ as of 2006 (PPP\$2,983) than in Australia (PPP\$3,445). ${ }^{26}$ Australia has proportionally greater numbers of practising health care professionals (including, doctors, general practitioners and midwives), more medical and nursing graduates, and notably higher rates of health workforce remuneration. ${ }^{26}$ Consequently, the Australian health system received a ranking of 32 out of 191 countries while NZ was ranked at $41.50,51$ The transTasman inequality between total number of healthcare workers, and remuneration of these workers, has substantial implications for the development of NZ's future healthcare workforce. NZ trains fewer doctors and an OECD-average number of nurses, but has one of the highest expatriation rates of medical staff, with about $23 \%$ of nurses and $29 \%$ of doctors leaving NZ. ${ }^{52}$ Not surprisingly, with greater investment in infrastructure and staff remuneration, Australia is the principal destination for NZ-trained medical staff. Building the health workforce capacity required to meet the increasing health demands of NZ's future older adult population will require a significant increase in expenditure on patient care and staff remuneration.

Government attention and public policy in both countries have emphasised cost, pursuing a neoliberal approach, while also attempting to promote health and well-being of their older and ageing populations..$^{53} \mathrm{It}$ is hoped that policy responses will be informed by evidence, such as presented here, with the politics of ageing a less significant driver in each country.

International data sources were used for population and health data. Standardised assumptions and methods are applied to population estimates from the United Nations, making comparisons across countries more reliable. Acknowledging the criticisms of some assumptions and modelling techniques, the Global Burden of Disease Study data and methods compile estimates of incidence, prevalence, and mortality from Australian and NZ data sources, correcting for known biases, to generate a summary measure comparable across countries. ${ }^{54,55}$ Understanding the limitations to these data, and that more recent data might be available, this metric was used nevertheless to try to minimise under- or overestimates of disease burden. Country-specific, age- and sex-disaggregated microdata files from the 2010 Global Burden of Disease Study are not available at the time of writing.$^{56}$ However, the overall results for the population aged 70+ years show slightly higher DALYs per 100,000 population in NZ than Australia, and a slightly higher contribution from non-communicable diseases to DALYs as a percentage of total DALYs. ${ }^{57}$ Cross-Tasman groups like ANZARC may in future provide an alternative to GBD estimates for older adult populations.

The domestic disability statistics in both countries are based on the International Classification of Functioning, Disability and Health (ICF), suggesting a high degree of comparability. ${ }^{58}$ The Australian Bureau of Statistics acknowledges the changes to the methodologies used to compile life tables for indigenous Australians that may impact the reliability of the life expectancy estimates. ${ }^{11}$ Life expectancy data for Māori are based on official estimates from Statistics NZ, CensusMortality Study. ${ }^{59}$

\section{Conclusions}

Policymakers and researchers everywhere are keen to predict the impacts of ageing on health and social services, labour force participation, income and retirement policies - then to compare the impacts of different policies and interventions. Australia and NZ have comparable median ages, and although there are differences in the rate and timing of population ageing between these countries in the shorter-term, projections for growth in the $60+$ and $80+$ populations are relatively parallel through 2100. Yet when examining disability and life expectancy, a contradictory story emerges needing further investigation, with higher healthy life expectancy for older Australian women compared to older NZ women and also higher disability levels.

Both countries have engaged actively and constructively on ageing, health and wellbeing through initiatives like the NZ Positive Ageing Strategy, and the Australian Advisory Panel on Positive Ageing and Ambassador on Ageing. It would be in the interest of both countries to have an active and dynamic cross-country, multi-disciplinary research collaboration, such as ANZARC, to highlight the modifiable processes of ageing and as a means to monitor and evaluate these initiatives and related policy. This paper, as part of a series, is "a shot over the ditch".

\section{Implications}

It is unclear whether age-related health and functioning declines in cohorts who are now in late-middle age will differ from those of the current older cohort in each country, or how the differing levels of health and disability will influence health care costs. Australia and NZ need to monitor trends, unravel the major population attributable risks, and identify preventive and other interventions that can stimulate and support lower age-related disability in their ageing populations.

\section{Acknowledgements}

This paper was submitted by the Australia/ New Zealand Ageing Research Consortium (ANZARC). The establishment of ANZARC was co-funded by grants from the Building Research Capacity in the Social Sciences (BRCSS) Network under their'Seeding Social Science Research Initiatives' portfolio, and from the College of Humanities and Social Sciences at Massey University. 
Researchers from the following projects were part of ANZARC meetings in 2010 and 2011:

New Zealand: The New Zealand Longitudinal Study of Ageing (NZLSA); Life and living in advanced age: A cohort study in New Zealand (LILACs NZ)

Australia:The Australian Longitudinal Study of Ageing (ALSA); Australian Longitudinal Study on Women's Health (ALSWH); Melbourne Longitudinal Surveys of Healthy Ageing (MELSHA); Ageing Babyboomers in Australia (ABBA); The Personality and Total Health (PATH) Through Life; Dynamic Analyses to Optimise Ageing (DYNOPTA)

WHO: Study on Global Ageing and Adult Health (SAGE); SAGE-INDEPTH

\section{References}

1. United Nations Statistics Division. Composition of Macro Geographical (Continental) Regions, Geographical Subregions, and Selected Economic and Other Groupings [Internet]. New York (NY): UN; 2013 [cited 2013 Sep 16]. Available from: http://unstats.un.org/unsd/methods/ m49/m49regin.htm

2. Department of Economic and Social Affairs. World Population Prospects: The 2012 Revision. New York (NY): United Nations Population Division; 2013.

3. Anderson G, Hussey P. Population aging: A comparison among industrialized countries. Health Aff. 2000;19(3):191-203.

4. Department of Economic and Social Affairs. World Population Ageing 2009. New York (NY): United Nations Population Division; 2009.

5. Organisation for Economic Co-operation and Development. Health Data 2012. Paris (FRA): OECD Publishing; 2012.

6. World Health Organisation. The Global Burden of Disease: 2004 Update. Geneva (CHE):WHO; 2008.

7. Australian Bureau of Statistics. 4125.0 - Gender Indicators. Canberra (AUST): ABS; 2012.

8. Ministry of Health. Tatau Kahukura: Māori Health Chart Book 2010. 2nd ed. Wellington (NZ): Government of New Zealand; 2010.

9. Ministry of Social Development. The Social Report 2010. Wellington (NZ): Statistics New Zealand; 2010.

10. Ministry of Health. Tatau Kura Tangata: Health of Older Māori Chart Book 2011. Wellington (NZ): Government of New Zealand; 2011.

11. Australian Bureau of Statistics. 3302.0.55.003 Experimental Life Tables for Aboriginal and Torres Strait Islander Australians. Canberra (AUST): ABS; 2009.

12. Smith GD. Epigenesis for epidemiologists: does evodevo have implications for population health research and practice? Int J Epidemiol. 2012;41:236-47.

13. Russell M, Nanayakkara B, Phillips D. The birthplace of chronic disease. A literature review. MSJA. 2012;4(1): 39-44.

14. Anderson I, Baum F, Bentley M, editors. Beyond Bandaids: Exploring the Underlying Social Determinants of Aboriginal Health. Proceedings of the Social Determinants of Aboriginal Health Workshop; 2004 July, Adelaide, South Australia. Darwin (AUST): Cooperative Research Centre for Aboriginal Health; 2007.

15. Reading CL, Wien F. Health Inequalities and Social Determinants of Aboriginal Peoples' Health. Ontario (CAN): National Collaborating Centre for Aboriginal Health; 2009.

16. Marmot M. Social determinants and the health of Indigenous Australians. Med JAust. 2011;194(10):512-3.

17. World Health Organization-Western Pacific and SouthEast Asia. Social determinants of health. Health in Asia and the Pacific. Manila (PHL):WHO; 2008.
18. Australian Bureau of Statistics. 4704.0. - The Health and Welfare of Australia's Aboriginal and Torres Strait Islander Peoples. Canberra (AUST): ABS; 2010.

19. Ministry of Health. The Health of New Zealand Adults 2011/12: Key Findings of the New Zealand Health Survey. Wellington (NZ): Government of New Zealand; 2012.

20. Zhao Y, Wright J, Begg S, Guthridge S. Decomposing Indigenous life expectancy gap by risk factors: a life table analysis. Popul Health Metr. 2013;11(1):1.

21. Ministry of Health. Health Loss in New Zealand:A Report from the New Zealand Burden of Diseases, Injuries and Risk Factors Study 2006-2016. Wellington (NZ): Government of New Zealand; 2013.

22. Davidson P, Digiacomo M, McGrath S. The feminization of aging: How will this impact on health outcomes and services? Health Care Women Int. 2011;32:1301-45.

23. Australian Bureau of Statistics. 4430.0. - Disability Ageing and Carers Australia: Summary of Findings. Canberra (AUST): ABS; 2011.

24. Statistics New Zealand. 2006 Disability Survey. Wellington (NZ): Government of New Zealand; 2007.

25. Lafortune G, Balestat G, the Disability Study Expert Group Members. Trends in Severe Disability among Elderly People: Assessing Evidence in 12 OECD Countries and Future Implications. Paris (FRA): Organisation for Economic Co-operation and Development; 2007.

26. Organisation for Economic Co-operation and Development. Health at a Glance 2011:OECDIndicators. Paris (FRA): OECD Publishing; 2011.

27. Denton FT, Spencer BG. Chronic health conditions: Changing prevalence in an aging population and some implications for the delivery of health care services. Can J Ageing. 2010;29(Special Issue 01):11-21.

28. Piazza J, Charles S, Almeida D. Living with chronic health conditions: Age differences in affective wellbeing. $J$ Gerontol B Psychol Sci Soc Sci. 2007;62(6):P313-21.

29. Economist Intelligence Unit. Healthcare Strategies for an Ageing Society. London (UK): The Economist; 2009.

30. Collerton J, Davies K, Jagger C, et al. Health and disease in 85 year olds: Baseline findings from the Newcastle 85+ cohort study. BMJ. 2009;399:b4904.

31. Crimmins EM, Beltrán-Sánchez H. Mortality and morbidity trends: Is there compression of morbidity? J Gerontol B Psychol Sci Soc Sci. 2011;66(1):75-86.

32. Fries JF, Bruce B, Chakravarty E. Compression of morbidity 1980-2011: A focused review of paradigms and progress. J Aging Res. 2011;2011:261702.

33. Statistics New Zealand. NewZealand Life Tables:2005-07. Wellington (NZ): Government of New Zealand; 2009.

34. Inglehart R, Foa R, Peterson C, Welzel C. Development, freedom, and rising happiness: A global perspective (1981-2007). Perspect Psychol Sci. 2008;3(4):264-85

35. Alley $D, C h a n g e V$. The changing relationship of obesity and disability: 1988-2004. JAMA. 2007;298:2020-7.

36. Bloom DE, Cafiero $\mathrm{E}$, Jané-Llopis $\mathrm{E}$, et al. The Global Economic Burden of Noncommunicable Diseases. Geneva (CHE):World Economic Forum; 2011.

37. Dormont B, Martins JO, Pelgrin F, Suhrcke M. The growth of health expenditures: Ageing vs. technological progress. In: Garibaldi P, Martins JO, van Ours J, editors. Ageing, Health and Productivity. Oxford (UK): Oxford Scholarship; 2010.

38. Graham P, Blakely T, Davis P, Sporle A, Pearce N. Compression, expansion or dynamic equilibrium? The evolution of health expectancy in New Zealand. J Epidemiol Community Health. 2004;58:659-66.

39. Gorman B, Read J. Gender disparities in adult health:an examination of three measures of morbidity. J Health Soc Behav. 2006;47(2):95-110.

40. Verbrugge L. The twain meet: Empirical explanations of sex differences in health and mortality. J Health Soc Behav. 1989;30(3):282-304.

41. Barford A, Dorling D, Davey Smith G. Life expectancy: Women now on top everywhere. BMJ. 2006;332(7545):808.

42. Tobias M, Blakely T, Matheson D, Rasanathan K, Atkinson J. Changing trends in Indigenous inequities in mortality: Lessons from New Zealand. Int J Epidemiol. 2009;38:1711-22.

43. Australian Institute of Health and Welfare. Indigenous Health [Internet]. Canberra (AUST): AlHW; 2013 [cited 2013 Sep 16]. Available from: www.aihw.gov.au/ indigenous-health/
44. Anstey KJ, Kiely KM, Booth $\mathrm{H}$, et al. Indigenous Australians are under-represented in longitudinal ageing studies. Aust N Z J Public Health. 2011;35(4): 331-6.

45. Kowal P, Gibson R, Wutzke S, et al. Roundtable discussion: Data on ageing Aboriginal and Torres Strait Islander populations. World Med Health Policy. 2011;3(3):1-11.

46. The 45 and Up Study. Validation of Aboriginal Identification Algorithms in the APDC and ABS Mortality Data Using the 45 and Up Study [Internet]. Sydney (AUST): The Sax Institute; 2010 [cited 2013 Sep 16] Available from: https://www.saxinstitute.org.au/ourwork/45-up-study/research-underway/\#validationof-aboriginal-identification-algorithms

47. Hayman K, Kerse N, Dyall L, et al. Life and living in advanced age: A cohort study in New Zealand -Te Puawaitanga o Nga Tapuwae Kia Ora Tonu, LiLACS NZ: Study protocol. BMC Geriatr. 2012;12(1):33.

48. Dulin PL, Stephens C, Alpass F, Hill RD, Stevenson B. The impact of socio-contextual, physical and lifestyle variables on measures of physical and psychological wellbeing among Māori and non-Māori: the New Zealand Health, Work and Retirement Study. Ageing Soc. 2011;31:1406-24.

49. Organisation for Economic Co-operation and Development (OECD). Society at a Glance 2009. OECD Social Indicators. Paris (FRA): OECD Publishing; 2009.

50. Organisation for Economic Co-operation and Development. Health Data 2008. Paris (FRA): OECD Publishing; 2008.

51. World Values Survey Association. World Values Surveys Wave 5 (2005-2008) [Internet]. New York (NY): World Values Survey Association; 2013 [cited 2013 Sep 16]. Available from: www.jdsurvey.net/jds/jdsurveyMaps. jsp? Idioma $=\mid \&$ SeccionTexto $=0404 \& \mathrm{NOID}=103$

52. Zurn P, Dumont J-C. Health Workforce and International Migration: Can New Zealand Compete? OECD Health Working Paper No.33. Paris (FRA): Organisation for Economic Co-operation and Development; 2008.

53. Fine $M$, Keeling $S$. Social policies for ageing societies: Australasian perspectives. In: Dannefer D, Phillipson C, editors. The SAGE Handbook on Social Gerontology. London (UK): SAGE Publications; 2010. p. 525-39.

54. Anand S, Hanson K. Disability-adjusted life years: a critical review.J Health Econ. 1997;16(6):685-702.

55. GBD Study Group. Global Burden of Diseases, Injuries and Risk Factors Operations Manual [Internet]. Seattle (WA): Institute for Health Metrics and Evaluation; 2009 [cited 2013 Sep 16]. Available from: http://health-equity. blogspot.com.au/2009/09/eq-global-burden-ofdiseases-injuries.html

56. GBD Study Group Global Burden ofDiseases, Injuries and Risk Factors Study 2010 [Internet]. Seattle (WA): Institute for Health Metrics and Evaluation; 2013 [cited 2013 Sep 16]. Available from: www.healthmetricsandevaluation. org/gbd/gbd-dissemination

57. GBD Study Group. Global Burden of Diseases, Injuries and Risk Factors Study 2010 [Internet]. Seattle (WA): Institute for Health Metrics and Evaluation; 2013 [cited 2013 Sep 16]. Available from: www.healthmetricsandevaluation. org/gbd/visualizations/gbd-cause-patterns

58. United Nations Economic and Social Commission for Asia and the Pacific. Training Manual on Disability Statistics. Bangkok (THA):UNESCAP; 2008.

59. Blakely T, Tobias M, Atkinson J, Yeh L-C, Huang K. Tracking Disparity: Trends in Ethnic and Socioeconomic Inequalitites in Mortality, 1981-2004. Wellington (NZ): Ministry of Health; 2007. 